\title{
REVISIONES
}

\section{Desde la distinción a la configuración: complejidad, evaluación y aprendizaje desde la visión relacional*}

\author{
From distinction to configuration: complexity, assesment and learning \\ from the relational approach
}

\section{Desde a distinção à configuração: complexidade, avaliação e aprendizagem do ponto de vista relacional}

\author{
Leonardo Lavanderos Gallardo', Iván Oliva Figueroa ${ }^{2}$ \\ ${ }^{1}$ Corporación SINTESYS \\ ${ }^{2}$ Universidad Austral de Chile, ivanoliva@uach.cl
}

\section{RESUMEN}

Dadas las actuales condiciones asociadas a la velocidad en que se producen las transformaciones a nivel global, lo que denominamos conocimiento ha comenzado a desfigurar la condición de certidumbre y determinabilidad con que fuimos conservadoramente formados. Este desfigurar ha impactado en la educación de manera tal que las epistemologías clásicas ya no tienen cabida para explicar cómo se propaga el aprendizaje en las redes. Sobre esta distinción, este trabajo propone una estrategia para poder evaluar el proceso de aprendizaje a partir de las condiciones de indeterminabilidad e incertidumbre.
\end{abstract}

Palabras clave: conocimiento, educación, teoría relacional, territorialidad, configuraciones.

\begin{abstract}
Given the current conditions attached to the speed at which changes occur globally, what we call knowledge has begun to misrepresent the condition of certainty and determinateness in which we were conservatively formed. This disfiguring education has made an impact on education making traditional epistemologies lacking space to explain how learning spreads across networks. On this distinction, this paper proposes a strategy to assess the process of learning from the conditions of indeterminacy and uncertainty.

Key words: knowledge, education, relational theory, territoriality, configurations.
\end{abstract}

\section{RESUMO}

Dadas as atuais condições associadas à velocidade em que as transformações se dão o que chamamos de conhecimento começou a desfigurar a condição de certeza e determinação a partir de onde fomos, conservadoramente, formados. Este desfigurar de tal condição tem impactado a propaga a aprendizagem em redes. Sobre esta distinção, propõese uma estratégia de avaliação do processo de aprendizagem a partir das condições de indeterminação e incerteza.

Palavras chave: conhecimento, educação, teoria relacional, territorialidade, configurações.

\footnotetext{
* El artículo integra el desarrollo epistemológico del Centro de Estudios en Teoría Relacional y Sistemas de Conocimiento, Corporación Sintesys y el trabajo de base en teoría de la complejidad y educación asociado al proyecto FONDECYT 1120863.
} 


\section{ANTECEDENTES GENERALES}

Gradualmente el fenómeno del conocer ha adquirido una centralidad explicativa de base en los modelos sociológicos y económicos a escala global. Ello se ha expresado en una importante diversidad conceptual asociada a nociones tales como sociedad del conocimiento, sociedad de la información, economías basadas en el conocimiento, sociedad de la incertidumbre, gestión del conocimiento, aprendizaje organizacional, entre otras. No obstante, pese a convertirse en uno de los axiomas más importantes de la sociología y economía contemporánea, el fenómeno del conocer sigue siendo trivializado desde enfoques reduccionistas, objetuales, lineales y sumativos. Esto último tiene implicancias fundamentales para el operar de los sistemas educativos, dado que holográficamente ${ }^{1}$ y a modo de una fractalidad semiótica, la educación en sus procesos de formación conlleva la presencia recursiva o huella matricial de la trama sociocultural que genera y a la que pertenece.

En este contexto, la problemática sociocultural se infiltra en el fenómeno educativo permeando sus principales lógicas de acción y legitimando estrategias de evaluación, en diversas escalas y dominios, que parecen privilegiar las magnitudes por sobre las formas de aprendizaje. Desde un enfoque relacional de la cognición, los procesos evaluativos en educación involucran más el cartografiado de configuraciones, que la medición de magnitudes cognitivas, en un tránsito desde el cuanto al cómo y por qué de todo campo de aprendizaje.

En tal sentido, los procesos evaluativos pueden desplazar sus premisas de base, transitando desde lógicas elementales referida a componentes y unidades cognitivas (datos, información adimensional) a lógicas configuracionales (formas, pautas y contextos semióticos), sustentadas en procesos de evaluación inspirados en una epistemología relacional. Ello trasunta en emergentes procesos de evaluación, construidos desde la base de estrategias cognitivas orientadas al mapeo de las diversas formas de comunicación en los procesos de enseñanza-aprendizaje, sus procesos de significación, equivalencia y co-generación de territorialidad.

\section{DE DOMINIOS ANALÓGICOS Y DISCRETOS DE LA COGNICIÓN}

En la perspectiva de Bateson (1984) y Spencer-Brown (1979) un universo nace cuando se secciona o se recorta un espacio, por lo que nuestra experiencia se deduciría inexorablemente del acto original de dicho recorte, en otras palabras, el acto epistemológico fundador de todo proceso cognitivo se sostiene en la capacidad de trazar una distinción. De este modo, los procesos primarios de la experiencia son las diferencias, en tanto contraste inmanente que permite generar las unidades básicas de todo sistema cognitivo,

1 La noción de propiedad hologramática hace alusión a la relación y co-producción entre niveles de observación globales y locales, en este caso, la relación sociedad - educación formal. Desde esta base, toda instancia global emerge de la organización de sus componentes locales, a la vez, toda localidad conlleva la presencia sistémica de la globalidad que la contiene. Los sistemas educativos, en estos términos, ya no pueden ser concebidos como sistemas espejo de la sociedad en la que se inscriben. 
esto es, la diferencia entre figura y fondo queda establecida en una mutua especificación, al menos, en términos binarios.

Derivado de lo anterior, la noción de distinción arriesga asumir un carácter discreto y sumativo, dado que el resultado de su actividad está abierto al recuento y la medición cuantitativa. No obstante, es fundamental reconocer que el establecimiento de dicho patrón elemental surge desde un metacontexto analógico o campo configurativo de sentido que posibilita tal distinción, en otras palabras, para constituir instancias discretas, (objetos, momentos, sucesos, etapas, etc.), es necesario además, un dominio analógico y configurativo que dé a la distinción su irrenunciable contexto de producción. Así planteado el proceso, la distinción aparece como una instancia discontinua y binaria (discreta) de la experiencia, en cambio, el campo de sentido y el patrón relacional de elementos que la posibilitó son esencialmente relacionales, continuos y configurativos (Oliva, 2010).

A partir de un modelo cognitivo minimalista, es posible describir al menos tres dimensiones inherentes a todo proceso cognitivo. a) una instancia elemental, referida a la diversidad de unidades derivadas de procesos de distinción; b) una instancia organizacional, referida a la configurabilidad relacional de dichas unidades y la emergencia de patrones de significación y c) una instancia contextual, referida al campo (espacio-tiempo-semiótico) emergente y productor de a) y b).

Desde esta base, podemos proponer que la generación de distinciones, en tanto instancia discreta de la experiencia, comporta lo analógico y configurativo en dos sentidos. Desde abajo la distinción deviene de un proceso relacional u organizacional de elementos, desde arriba, la distinción deviene de un metacontexto que da un campo de sentido para que éstas surjan. La distinción, de este modo, queda atrapada en todas sus fronteras por un universo relacional y continuo de carácter analógico. De este modo, los dominios desde donde emergen y donde recaen las distinciones, difícilmente pueden responder a lógicas acumulativas, sino más bien a lógicas configurativas en constante transformación y fuga. Aunque la noción de distinción comporta en su génesis aspectos discretos y binarios, como la diferencia, el trazado y el contraste, la distinción es una unidad relacional que emerge de la actividad de un bucle discreto $\leftarrow \rightarrow$ analógico (Oliva, 2010).

En este contexto, una distinción es una acción que consiste en una configuración de diferencias, en otras palabras, el sistema cognitivo en la distinción señala la unidad, ejecutando la operación que define sus límites, separándola respecto de un trasfondo. De este modo, dado el énfasis en la relación, la información es adimensional y no cuantificable, ya que la diferencia lo es, en tanto es inmanente a dicha relación (Lahitte et al. 1989). Del mismo modo, la diferencia, no localizable ni cuantificable, emerge relacionalmente, lo que implica su irreductibilidad. Así, el conocimiento se hace en la posibilidad de producir semiosis de la diferencia configurándola de manera recursiva y por ende evolutiva.

En síntesis, al asumir el carácter irreductible del aprendizaje y sus procesos de significación, asumimos también que toda dinámica cognitiva se manifiesta por patrones integrados y sistemas eco-auto-organizados de distinciones, no reducibles a las lógicas disyuntivas, sumativas y/o secuenciales en la que descansan hegemónicamente los procesos evaluativos en la educación formal. Este supuesto da al fenómeno del conocer una óptica fundamentalmente relacional, enfatizando en la mutua producción de las instancias discretas $\leftarrow \rightarrow$ analógicas del aprendizaje y sus emergentes campos de indeterminabilidad e incertidumbre (Figura 1). 


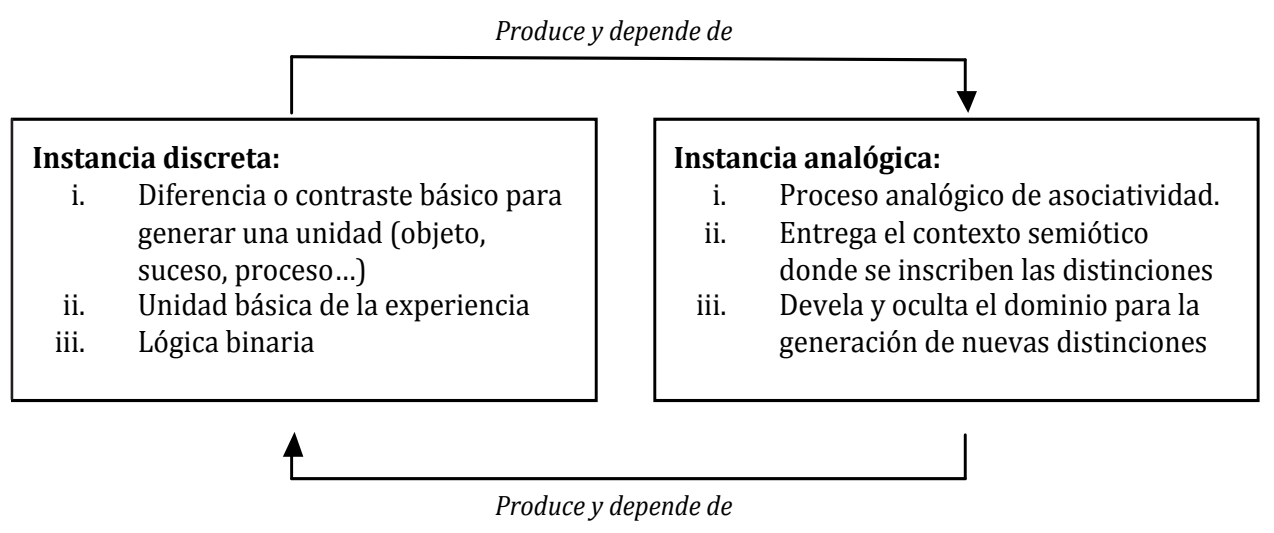

Figura 1. Bucle conformado por las instancias discretas y analógicas del aprendizaje (Oliva, 2010)

\section{DESDE LA TRIFERENCIA A LA CONFIGURACIÓN DE MUNDO}

Desde la base de este entendimiento, hemos denominado aproximación relacional del conocer a la posición epistemológica que privilegia la unidad relacional observador-entorno (culturador) como proceso de construcción de territorialidad. Definiendo territorialidad como proceso de equivalencia efectiva-afectiva en el intercambio de mapas o paisajes (configuraciones de significado), a partir de la actividad generada en los entornos de observadores en comunicación. Desde esta base, la efectividad emerge en el dominio de lo afectivo (Lavanderos, 2002).

El conocer, desde esta visión, queda definido como un proceso emergente de configuraciones relacionales que son fundadas desde la generación de diferencias de un observador que entorna y que sólo tiene significado para él (Lavanderos y Malpartida 2001, Malpartida, 1991, Malpartida y Lavanderos, 1995, 2000). Este significado es lo que permite agenciar pautas de territorialidad o, dicho de otra manera, generar identidad desde el agenciamiento y la pertenencia. A partir de este proceso, la territorialidad como idea colectiva es co-construida entre los observadores que constituyen la red. En consecuencia, las descripciones e interpretaciones se determinan a través de mecanismos internos de comunicación (clausura comunicacional) lo que definiremos como generación de configuraciones de territorialidad.

Por lo tanto, desde esta visión la territorialidad no es experienciable como objeto físico, sino como la estrategia de selección de alternativas de elementos descriptivos que emerge, como propiedad constitutiva de la relación de observación (Abel 1998, Bateson 1984, Bullen et al.1997, Edmonds 1996, Heylighen 1997, Varela et al., 1992). Desde esta perspectiva, el proceso descriptivo-interpretativo no se aplica a un territorio, sino que es un proceso de co- $\neg$ circunstancialidad en la distinción de unidades, puesto que implica tanto la definición del observador como la definición de la unidad observada.

Derivado de lo anterior, el observador se constituye en el acto de distinción como unidad (Maturana y Varela, 1984), siendo centralizador de la relación con lo observado y por lo tanto participante de ello. Desde la escuela relacional, podríamos resumir el proceso cognitivo como: la generación de configuraciones de distinciones, en relación con el 
significado del intercambio de éstas, producto de la territorialidad del observador. La territorialidad del observador se evidencia desde su operar discriminativo-afectivo (distinción), en relación con la unidad de observación, el cual por algún criterio corta una secuencia y la expone actuando sobre la base de algún significado (que debe ser explicado).

En este contexto, la posibilidad de describir surge de nuestra historia de descripciones, de nuestra cultura, reconociéndonos como parte del sistema de observación implicado en la trama comunicacional. Desde esta perspectiva, la configuración de territorialidad es co-construída a partir de nuestras distinciones como proceso relacional cultura-naturaleza. El observador ya no puede ser considerado sólo como autonómico, esto es, que responde a mecanismos internos de autoorganización (Varela et al., 1992) sino como eco-semioautonómico, esto es, que se reproduce en relación a partir de la producción semiótica. En este contexto, la observación como forma de distinción no sólo se construye a partir de ciertos criterios que es necesario explicitar, sino que además responde a una estrategia y necesariamente a un estilo cognitivo (Maruyama, 1980). Recordemos que en ellos cobra vital importancia la comunicación entre los observadores, para quienes los mensajes tienen un significado que se encuentra determinado por la historia de relaciones y comunicaciones previas. Las clasificaciones, las jerarquías y, por último, la organización, emergen como parte del proceso de conservación de la relación cultura-naturaleza, es decir no se aplican sobre algo.

La relación es la base por la cual y, sobre la cual, decimos que como observadores, extraemos diferencias y que esas diferencias extraídas de la relación son argumentadas como distinciones. En rigor es una triferencia, dado que el proceso implica al que genera. En ese proceso, la información es la primera noticia de la triferenciación. Lo anterior nos lleva a plantear que todo proceso cognitivo ocurre en una entidad capaz de generar triferencias y procesarlas como información. La información así generada puede luego ingresar en el dominio de la comunicación humana con su enunciación (configuración de mundo), la que para el interlocutor toma la forma de un mensaje. Bateson (1984) escribió que la información era el producto de una diferencia que hace una diferencia en un momento posterior. En este proceso pues, se encuentran unidos en una sola función el referente y el observador-entorno.

Lo que se conoce como relaciones son los emergentes de múltiples distinciones que los observadores en su entorno de observación generan. Siendo esto así, son también múltiples las calificaciones que la relación de base puede recibir. Nuestro lenguaje es objetual, y en el proceso continuo de sustantivación, hemos transformados verbos en sustantivos. La relación, lo relacional, debe ser entendido como proceso-functor o metavinculador y no como un objeto, de hecho se enseña que los verbos predican de acciones y pocas veces que son vínculos entre el sujeto y el predicado.

La diferenciación primaria de la relación es la triferencia y su argumento una distinción (información). Desde la relación cognitiva, lo primario consiste en las distinciones, reconocer como distinto a lo que rodea. En tal sentido, la distinción predica de la capacidad de recortar, circunscribir una unidad y separarla del resto. La extracción de una unidad, la distinción figura/fondo tiene que ver con la individualización y no necesariamente con que éstas sean diferentes de hecho ya sea genéricamente o específicamente.

Las sucesivas triferencias forman los objetos determinados culturalmente para ser distinguidos como distintos del observador, de esta manera ingresan en el proceso de las sucesivas diferenciaciones que forman el espiral de lo distinto-semejante. En síntesis el 
proceso de conocimiento puede ser explicado como el tránsito desde la producción de triferencias hasta sus niveles más complejos que son secuencias que permiten narrar y configurar un mundo donde si es pre-dado o dado resulta irrelevante.

A la base de lo expuesto, el proceso de educación requiere de aproximaciones epistemológicas que permitan operar desde conceptos relacionales. Lo anterior se traduce en que para un sistema dado como eco-semio-auto-organización, la cultura determina activamente el arreglo de sus componentes y la significación de su carácter, o de su comportamiento, lo que es significativo solamente con respecto a sí misma. Este operar genera la trama de distinciones de su propia diversidad y conectividad. Desde estas perspectivas, la educación como proceso toma lugar en el espacio relacional de la comunicación, lo que significa que nuestra condición de observadores-decidores se experiencia en la forma de relacionarnos unos con otros para generar sentido de agenciamiento y pertenencia.

Entonces, la reproducción de un sistema determinado culturalmente sólo es posible, desde esta perspectiva, dentro de una red semiótica. Por lo tanto, una configuración de territorialidad se constituye como parte de esta red cuando los miembros de la cultura la agencian y la realizan al vivirla. Como tal, la identidad y complejidad de una cultura, surge continuamente cuando ellos viven culturalmente la territorialidad que ellos integran. Esta vivencia es lo que denominamos educación afectiva-efectiva.

\section{CULTURA Y EDUCACIÓN DESDE LA VISIÓN RELACIONAL}

Previo a su definición, es necesario develar los conceptos canónicos sobre los cuales descansa esta epistemología, así como describir el proceso que explica nuestra epistemología de base.

Desde la perspectiva relacional del conocimiento (Lavanderos et al., 2001) definiremos cultura como la poiesis de meta-configuraciones (pautas, patrones o prácticas) de agenciamiento (lo que uno hace suyo) y pertenencia (uno se hace parte de) organizadas de manera de lograr conservar la organización relacional. Este proceso es al que denominamos Territorialidad (Lavanderos et al., 2001). Asimismo, definiremos proceso educativo como la estrategia guiada de acoplamiento entre las decisiones estratégicas, que hacen posible el manejo de los procesos y las relaciones que generan territorialidad.

Todo lo dicho anteriormente implica que las operaciones de distinción son configuraciones pautadas por redes de observadores, lo que implica que sus formas y tipos sólo pueden ser entendidas como meta configuraciones organizadas a partir de la conservación y producción de esas pautas. Por lo mismo, si una distinción implica al configurador que la opera, el proceso descriptivo descansa en ese operar afectando a lo observado de modo tal que impiden toda creencia predictiva. Por lo tanto, podemos afirmar que este operar sólo puede ser comprendido a partir del cómo generamos las distinciones (von Foerster, 1974).

Abordar el fenómeno del conocer desde un enfoque relacional y configuracional es, a la vez, abordarlo en su dimensión paradigmática, enfatizando en sus instancias de disyunción, asociación, jerarquización y centralización. Desde esta perspectiva, para entender y evaluar la propagación del aprendizaje en las redes, es necesario concebir todo proceso cognitivo al menos en tres dimensiones: a) la forma u organización paradigmática de 
determinadas instancias cognitivas, b) las redes comunicacionales emergentes productoras y productos de a), y c) las implicancias éticas-estéticas de estos procesos configuracionales (Oliva, 2006).

\section{LA EVALUACIÓN DEL APRENDIZAJE DESDE LA VISIÓN RELACIONAL}

Todas las actividades humanas son operaciones semióticas. De esta manera, las unidades generadas como operaciones de distinción pueden ser explicadas a partir de la estructura semiótica. Lo anterior se refiere al tipo y número de relaciones que un observador produce entre los conceptos que utiliza en el proceso explicativo.

El éxito del proceso educativo, definido anteriormente, depende fundamentalmente de la coherencia entre lo que se describe, la explicación asociada y la legitimidad de la tautología dentro de la red relacional. La descripción de las acciones no soporta ninguna lógica, es, como señala Bateson (1984) una serie de hechos de los que no sabemos cómo se interconectan. Por lo mismo, la explicación no suministrará ninguna información más que la que ya posee la descripción. Es entonces cuando la tautología o la forma conectiva aplicada a la descripción permiten la conexión de las acciones generando sentido a la serie de hechos contenidos en la descripción de un determinado contexto. Entonces, cuando nos referimos a la legitimidad de la tautología, lo que afirmamos es que para una red de relaciones, un discurso educativo no necesariamente logra una explicación de la generación de coherencia en la toma de decisiones; esto significa que la narrativa formativa, para ser efectiva-afectiva, debe coincidir con la sostenibilidad de la red, esto es, la legitimidad de las tautologías utilizadas en el proceso de educación.

Cuanto mayor es la legitimidad tautológica, mayor será la coherencia en el proceso formativo, lo que tendrá como consecuencia una red relacional altamente cohesiva, coordinada, descentralizada y con alto poder de cambio. Entonces, el proceso de sostenibilidad de la red a partir de la educación implica el intercambio efectivo semiótico-estético que le permite a ésta actuar coherentemente para reproducirse. Entendemos semiótica-estética como la generación de configuraciones relacionales efectiva y afectiva de pertenencia y agenciamiento. Lo anterior puede ejemplificarse de la siguiente manera: no es suficiente que el plan de formación genere la orientación de las acciones con alto valor explicativo, fruto de la tautología aplicada, sino que también debe ser legitimada en los afectos o confianza de los que viven el plan.

\section{APRENDER: DE LA MEDICIÓN A LA CARTOGRAFÍA}

La caracterización estructural se basa en la aproximación saussuriana de ejes de relaciones sintagmáticas y paradigmáticas (Lahitte, 1987). Estas relaciones sintagmáticas se relacionan con la presencia de términos o palabras en una serie cualquiera; es lo que mencionamos anteriormente como la descripción de acciones, mientras que las relaciones paradigmáticas unen términos o palabras sin precisar una forma particular para dicha unión. El eje paradigmático de un discurso traduce relaciones esenciales, estables e implícitas. Este eje es el que permite evidenciar la tautología. 
Dentro de la perspectiva del relativismo cultural, se define claramente un campo en el cual hay un número de asociaciones paradigmáticas, las cuales no necesitan ser dichas. Estas asociaciones pueden ser objeto de desarrollos explícitos en otros lugares y en otras épocas. Ello por cuanto son propios de cada cultura, involucrando a todos los aspectos del conocimiento, incluido el científico. La caracterización estructural del discurso continúa con el de analogías entre los ejes del discurso, las distinciones y la relacionalidad utilizada. Ello se realiza explicitando el sintagma discurso, que corresponde a las distinciones a partir de una pregunta base, así como el paradigma pensamiento, el cual establece la red de asociaciones entre las distinciones y el tipo de asociaciones utilizadas, sean éstas asociativas o causales. Algunas reglas o pautas que permiten conectar los sintagmas pueden ser las de consecución y asociación, en el cual la consecución corresponde a conceptos en los cuales la presencia de uno afecta al otro y su conexión es temporal. Dentro de ellos, el esquema más simple es el de causalidad. La asociación, por su parte, corresponde a conceptos que superponen parte de sus significados en su relación.

De lo anterior se establece que el proceso de evaluación del aprendizaje queda modelado como un conjunto de conceptos consecutivos y asociativos desde su base de distinciones. Sin embargo, en la abducción no importan las unidades (sintagmas) que componen una descripción particular, sino la formalidad de sus relaciones, lo que permite la emergencia de determinada forma explicativa. Así, lo fundamental en el proceso de reformulación de una pregunta es si la configuración relacional de los sintagmas permite el tránsito de la reformulación a la explicación.

El método específico que se usa para este tipo de modelación, se basa en el concepto de mapas cognitivos. Este corresponde a un sistema computacional que grafica la línea argumental del observador, explicitando sus conceptos y las conexiones que establece entre ellos. Mediante el análisis de la estructura graficada se pueden conocer los atractores o centralizadores del discurso, tautologías, grados de completitud, elementos terminales, elementos iniciadores, etc. A partir de este tipo de cualidades, es posible distinguir que algunos conceptos del plano argumental centralizan y pautan la conectividad entre las ideas y conceptos utilizados en la explicación. Más importante aún, el mapa cognitivo explicita el marco o paradigma desde donde el observador construye su observación, permitiendo estructurar y analizar diferentes líneas argumentales. El mapeo cognitivo se desarrolla directamente a partir de entrevistas, permitiendo que los observadores construyan para un contexto determinado por una pregunta una configuración entre sintagmas y paradigmas.

Lo que denominamos conocimiento implicaría configuraciones de base, las cuales en un primer momento quedan plasmadas en el mapa. El mapa explicitaría, mediante la interpretación de su estructura, el nivel de complejidad de un concepto. Desde esta perspectiva, la relación enseñanza-aprendizaje sería explicita ya que el docente o educador debe señalar la configuración que desea territorializar.

La hipotésis que subyace a la afirmación anterior, es que si se produce el proceso de educación, el mapa inicial cambia y se hace equivalente al mapa del educador. Es importante que la equivalencia no se confunda con igualdad, ya que sería un acto de repetición y no un acto de acoplamiento. Así la evaluación del aprendizaje se grafica como diversidad organizacional entre mapa cognitivos, a partir de conceptos, así como de sus conexiones. 
El proceso de aprendizaje puede ser evaluado, como señalamos anteriormente, a partir de los cambios en complejidad del mapa. Nos referimos a complejidad cuando la unidad gráfica no es trivial ni susceptible de dividir o reducir a unidades simples dado la configuración de conexiones. En este contexto, es posible distinguir a lo menos cinco dominios constitutivos de los mapas cognitivos asociados a procesos de aprendizaje. Cabe señalar que la posibilidad de generar estrategias de evaluación en todos estos dominios, prescinde de la convergencia cognitiva de las instancias elemental, organizacional y contextual.

\begin{tabular}{|l|l|}
\hline Dominio & Descriptor \\
\hline $\begin{array}{l}\text { Número y tipo de } \\
\text { distinciones }\end{array}$ & $\begin{array}{l}\text { Si el proceso de aprendizaje fue efectivo el mapa aumenta su } \\
\text { poder explicativo logrando develar procesos abductivos y } \\
\text { diversidad de distinciones }\end{array}$ \\
$\begin{array}{l}\text { Completitud y densidad de circuitos } \\
\text { relacional } \\
\text { reducen a causas y efectos. }\end{array}$ & $\begin{array}{l}\text { Las líneas argumentativas soportan todo el proceso } \\
\text { reformulativo. No se observan dominios de aprendizaje } \\
\text { fragmentados }\end{array}$ \\
Centralización & $\begin{array}{l}\text { Evaluación de los principio explicativo asociados al } \\
\text { aprendizaje. Pueden ser clasificados de alta o baja } \\
\text { certidumbre } \\
\text { Tipos de conectividad }\end{array}$ \\
$\begin{array}{l}\text { Tipo cognitivos, causales y asociativos. Permite evaluar } \\
\text { flexibilidad en los procesos de aprendizaje }\end{array}$ \\
\hline
\end{tabular}

Tabla 1. Características organizacionales del mapa cognitivo asociados al proceso de aprendizaje

\section{CONCLUSIONES}

Derivado de lo señalado precedentemente, la noción de organización no es trivial, dado que significa asumir una perspectiva de evaluación orientada a los patrones de significación y pertinencia de las formas de conocer generadas hegemónicamente por los sistemas de educación formal. Desde esta base, la organización del conocer humano asume un dominio no estructural, transformándose en una tarea a la vez capital, aleatoria e incierta, lo que requiere pensar lo educativo de forma radicalmente compleja (Morin, 1991). En consecuencia, las estrategias de evaluación desde una visión relacional, asumen que el fenómeno del conocer, por su carácter complejo y relacional, resiste todo proceso de determinación, disyunción, unidimensionalización y cuantificación trivial.

En este contexto, resulta imprescindible constituir el proceso educativo como un arte de la relación, esto es, que no existen dentro de los diferentes tipos de conceptos límites o fronteras. El conocimiento es finalmente un recrear de prácticas y pautas que 
consiguen que lo ético-estético cohesione desde lo afectivo-efectivo. Educar es permitir volver al mundo inicial del juego de crear a la madre y al padre, un juego relacional, de otra manera, un acto lúdico sin fronteras. El mapa es entonces este proceso lúdico donde plasmamos en la cartografía nuestras conexiones cotidianas, las cuales construyen los paisajes que permiten la toma de decisiones y que sin cuestionarlas las hacemos parte de nuestro configurar para navegar dentro de redes.

Desde una visión relacional, la premisa de Lord Kelvin: Todo cuanto existe, existe en una cantidad y por ende puede ser medido, no es solo la manifestación de una profunda patología epistémica, sino una condicionante nodal para el desarrollo de esquemas evaluativos inspirados por una visión más compleja del aprendizaje, sus mediaciones y formas de distribución en la redes.

\section{REFERENCIAS BIBLIOGRÁFICAS}

Abel, T. (1998). Complex Adaptive Systems, Evolutionism, and Ecology within Anthropology: Interdisciplinary Research for Understanding Cultural And Ecological Dynamics. Georgia Journal of Ecological Anthropology, vol. 2, 6-29.

Bateson, G. (1984). Pasos hacia una ecología de la mente. Buenos Aires: Carlos Lohlé.

Bullen, N, Jones, K. y Duncan, C. (1997). Modelling complexity: Analysing between-individual and between-place variation - a multilvel tutorial. Environment and Planing A, n. 29, 585-609.

Edmonds, B. (1996). What is Complexity. En F. Heylighen \& D. Aerts (eds.), The Evolution of Complexity. Dordrecht: Kluwer.

Heylighen, F. (1997). Evolution and Complexity: an introduction to the book. En F. Heylighen \& D. Aerts (eds.), The Evolution of Complexity. Dordrecht: Kluwer.

Lahitte, H; Hurrel, J; y Malpartida,A. (1987). Relaciones 1: De la Ecología de las Ideas a la idea de la Ecología. Buenos Aires: MAKO.

Lahitte, H.Hurrel,J y Malpartida, A. (1989). Relaciones 2: Crítica y expansión de la Ecología de las Ideas. Buenos Aires: Nuevo Siglo.

Lavanderos, L. (2002). La organización de los Sistemas Cultura-Naturaleza. Tesis Doctoral. Santiago: Facultad de Ciencias, Universidad de Chile.

Lavanderos, L; Malpartida, A. (2001). Cognición y Territorio. Santiago: Editorial Universitaria UTEM.

Malpartida, A. (1991). La noción de entorno en etología (una discusión etimo-epistemológica). Ecognición, vol. 2, n. 1, 39-46.

Malpartida, A; Lavanderos, L (1995). Una aproximación sociedad-naturaleza. El Ecotomo. Revista Chilena de Historia Natural, n. 68, 419-427.

Malpartida, A; Lavanderos, L (2000). Ecosystem and Ecotomo:a nature or society-nature relationship? Acta Biotheoretica, vol. 48, n. 2, 85-94.

Mayurama, M. (1980). Mindscapes and Science Theories. Current Anthropology, n. 21, 589-608.

Maturana, H; Varela, F. (1984). El Árbol del Conocimiento: Las Bases Biológicas del Entendimiento Humano. Santiago: Editorial Universitaria.

Morin, E. (1991). Introducción al Pensamiento Complejo. Barcelona: Gedisa.

Oliva, I. (2007). Conocimiento y Complejidad: Aportes para una Paradigmatología de lo Educativo. Revista Estudios Pedagógico, vol. 33, n. 1, 109-128.

Oliva, I. (2010). Breve cartografía de una disonancia epistémica: educación, complejidad y reforma. Polis, Revista de la Universidad Bolivariana, vol. 25, 321-335.

Spencer Brown, G. (1979). Laws of Form. New York: E.P. Dutton. 
Varela, F; Thompson, E; Rosch, E. (1992). De cuerpo presente. Barcelona: Gedisa.

von Foerster, H. (1974). Cibernetics of cibernetics. Illinois Biological Computer Laboratory, Univ. of Illinois. 
